\title{
Las políticas de desarrollo rural en Costa Rica: Avances y desafíos desde las perspectivas del territorio
}

Recibido: 12 de noviembre 2019 Revisado: 23 de noviembre 2019

Apufasabarborzarzarpas

Costarricense. Máster en

Gerencia del Comercio

Internacional. Centro

Internacional de Política

Económica, Universidad

Nacional, Costa Rica. Investigador del CINPE-UNA. Sus temas de interés incluyen la sociología rural, la creación de

capacidades locales para el desarrollo rural y los sistemas de innovación agropecuaria y rural.

Correo electrónico:

luis.barboza.arias@una.ac.cr

\section{Alejandro Rodríguez Miranda}

Costarricense. Estudiante de

Bachillerato en Economía.

Universidad Nacional, Costa

Rica. Asistente de investigación

del CINPE-UNA, Costa Rica. Sus

temas de interés incluyen la

competitividad territorial, los

sistemas de gobernanza

territorial y el desarrollo

territorial rural. Correo electrónico:

alejandro.161296@hotmail.com

Fernando Sáenz Segura Costarricense. Ph.D. Universidad de Wageningen. Catedrático del

CINPE, Universidad Nacional,

Costa Rica. Sus temas de interés incluyen la economía

institucional, el análisis de políticas públicas y el desarrollo de territorios rurales. Correo electrónico:

fernando.saenz@una.ac.cr
Resumen: En este estudio se lleva a cabo una revisión y caracterización de los Planes Nacionales de Desarrollo en Costa Rica, con base en la evolución político-institucional de las administraciones Solís Rivera (2014-2018) y Alvarado Quesada (2018-2022). El objetivo del artículo es analizar la incorporación de la perspectiva territorial al diseño de políticas públicas de desarrollo rural y su evolución en el marco de los instrumentos dirigidos a promover el desarrollo sostenible de los territorios rurales. Algunas preguntas específicas que se plantean son las siguientes: ¿cómo estas políticas dieron origen a instrumentos específicos para atender la complejidad e interseccionalidad de los problemas rurales? ¿Cuáles son los efectos de estas políticas e instrumentos en el desarrollo sostenible de los territorios rurales de Costa Rica? ¿Cuáles son los desafíos de una agenda complementaria de desarrollo rural con enfoque territorial?

Palabras clave: desarrollo económico y social; elaboración de políticas; estrategia de desarrollo; planificación de programas; planificación rural

\section{Rural development policies in Costa Rica: advances and challenges from territorial approach}

Abstract: This paper is a review of the last two National Development Plans, implemented by the Central Government of Costa Rica. We study the political and institutional evolution of Solís Rivera's (2014-2018) and Alvarado Quesada's (2018-2022) administrations. The objective of the research is to analyze the territorial perspective into public policies for rural development design, and their evolution as part of the instruments aimed at promoting the sustainable development of rural territories. Some specific questions are: how these policies gave rise to specific instruments to address the complexity and intersectoriality of rural problems? What are the effects of these policies and instruments on the sustainable development of rural territories in Costa Rica? What are the challenges of a complementary rural development agenda with a territorial approach?

Key words: economic and social development; development strategies; policy making, programme planning; rural planning 


\section{Introducción}

\section{Contexto y objetivo del artículo}

En Costa Rica, una discusión pública sobre los cambios y desafíos que enfrenta el desarrollo rural tomó forma en el contexto de la campaña política que culminó en las elecciones presidenciales de 2018, cuya segunda vuelta, realizada el $1^{\circ}$ de abril, dio como vencedor al candidato del Partido Acción Ciudadana (PAC, el partido oficialista), Carlos Alvarado Quesada.

En este sentido, resulta oportuno llevar a cabo un ejercicio de reflexión académica en el que se problematice dónde estamos con respecto al nivel de avance y cuál es el estado actual de las políticas de desarrollo rural, considerando la incorporación del enfoque territorial. La pregunta de investigación principal a la que se busca responder es: ¿cómo se ha integrado la variable territorial al diseño de políticas públicas de desarrollo rural en ambas administraciones? Algunas preguntas específicas que se plantean son las siguientes: ¿cómo estas políticas dieron origen a instrumentos específicos para atender la complejidad e intersectorialidad de los problemas rurales? ¿Cuáles son los efectos de estas políticas e instrumentos en el desarrollo sostenible de los territorios rurales de Costa Rica? ¿Cuáles son los desafíos de una agenda complementaria de desarrollo rural con enfoque territorial?

El objetivo del artículo es, por lo tanto, analizar la incorporación de la perspectiva territorial al diseño de políticas públicas de desarrollo rural, considerando la actual administración, Alvarado Quesada (2018-2022) y la anterior Solís Rivera (2014-2018), y su evolución en el marco de los instrumentos dirigidos a promover el desarrollo sostenible de los territorios rurales de Costa Rica.

La estructura del documento se divide en cuatro secciones. Luego del apartado introductorio, se presenta la metodología utilizada. También se incorporan elementos teóricos y conceptuales, útiles para el planteamiento y la descripción del caso. En la segunda sección, se describen los planes de desarrollo en estudio. Se procede a la discusión de resultados en la tercera sección. En la cuarta sección, se concluye y se establecen algunos insumos para la reflexión posterior sobre el tema.

\section{Metodología}

Este artículo forma parte de una actividad de investigación realizada en el Centro de Internacional de Política Económica para el Desarrollo Sostenible, de la Universidad Nacional de Costa Rica (CINPE-UNA), cuyo propósito principal es ofrecer una primera caracterización de la incorporación del enfoque territorial del desarrollo rural en el diseño de instrumentos de política vinculados a los Planes Nacionales de Desarrollo de las administraciones Solís Rivera (2014-2018) y Alvarado Quesada (2018-2022). Con esa finalidad, se lleva a cabo la revisión y caracterización de ambos planes, a partir del estudio 
de la evolución político-institucional. Los resultados preliminares de este artículo fueron presentados en el V Foro bienal de Estudios del Desarrollo, organizado por la Red Iberoamericana de Estudios del Desarrollo (RIED), en mayor de 2019, en Bogotá, Colombia.

La decisión metodológica de seleccionar este periodo para la realización del estudio se tomó después de considerar a la publicación, y posterior entrada en vigor, del Programa Tejiendo Desarrollo, propuesto por la administración Solís Rivera (2014-2018), como un antecedente institucional importante en el diseño de instrumentos de políticas con enfoque territorial en el país.

En él, se menciona por primera vez y de manera explícita los efectos de la desigualdad territorial en el desarrollo y el acceso a oportunidades; asimismo, se identifican acciones claras para promover el fortalecimiento de los marcos de gobernanza territorial y la participación de los actores locales en procesos de coordinación y diseño de programas y proyectos regionales, territoriales y cantonales.

Tejiendo Desarrollo tiene como pilares los principios de pluralidad e inclusión, ética y transparencia, con respecto a la autonomía municipal y el fortalecimiento de la institucionalidad (Despacho de la Primera Dama, 2015). Además, es importante mencionar que, en conjunto con la Estrategia nacional de empleo y producción, representa un referente principal en el diseño de la Estrategia para la atención de la pobreza y la reducción de la pobreza extrema: Puente al desarrollo, que define como área de interés prioritario el abordaje integral y la incidencia en los territorios y gobiernos locales (IMAS, 2015).

El abordaje en este artículo es cualitativo, descriptivo y analítico, con la finalidad de ofrecer una primera revisión del tema, en función de la pregunta de investigación planteada. En la recolección de la información se mezclaron fuentes primarias y secundarias. En el primer caso, la recolección se realizó por medio de una entrevista semiestructurada con Harys Regidor Barboza, presidente ejecutivo del Instituto de Desarrollo Rural (INDER). Se utilizó una guía de entrevista, con la cual se pidió la opinión del informante sobre tres grandes temas: 1) la evolución y características de las políticas de desarrollo rural; 2) grado conocimiento institucional y grado de incorporación del enfoque de desarrollo territorial rural; y 3 ) identificación de los principales cambios institucionales y el nivel de avance en cuanto a la agenda de desarrollo rural.

El análisis de la evolución de las políticas para el sector agropecuario y los territorios rurales tuvo las tres fases: 1 . Matriz - inventario de los principales instrumentos de política identificados en los Planes Nacionales de Desarrollo estudiados; 2. Evaluación cualitativa: percepción sobre resultados esos instrumentos actuales por parte de la presidencia ejecutiva del INDER; 3. Sistematización del gasto nacional público agropecuario y análisis de la evolución de los indicadores de pobreza y desempleo rural. 


\section{Fundamentos teóricos y conceptuales del tema}

Para entender el marco institucional y de diseño de las políticas públicas para el desarrollo rural se utilizan algunos axiomas de la sociología de la acción pública (Fontaine 2015,6 ). La sociología de la acción pública incorpora una serie de categorías para el análisis del diseño de políticas públicas, que van más allá de la lógica de análisis estadocéntrica. Las categorías utilizadas en este estudio se explican en el cuadro 1.

\section{Cuadro 1. Categorías de la sociología de la acción pública utilizadas en el} análisis del estudio de caso

\begin{tabular}{|c|c|c|}
\hline Nombre de la categoría & Definición conceptual & Definición operativa \\
\hline Información & $\begin{array}{l}\text { Instrumentos que } \\
\text { producen datos para el } \\
\text { gobierno y a los datos } \\
\text { producidos por este último }\end{array}$ & $\begin{array}{l}\text { Documentos de políticas } \\
\text { dirigidas al sector rural y } \\
\text { agropecuario. Planes } \\
\text { Nacionales de Desarrollo. } \\
\text { Memorias institucionales. }\end{array}$ \\
\hline Autoridad & $\begin{array}{l}\text { Normas jurídicas y } \\
\text { sistema legal que } \\
\text { enmarcan las } \\
\text { políticas }\end{array}$ & $\begin{array}{l}\text { Marco legal y legislación } \\
\text { que rige la } \\
\text { institucionalidad rural y } \\
\text { agropecuaria en Costa } \\
\text { Rica. }\end{array}$ \\
\hline Tesoro & $\begin{array}{l}\text { Instrumentos fiscales, } \\
\text { financieros y monetarios } \\
\text { que las hacen viables o } \\
\text { las obstaculizan }\end{array}$ & $\begin{array}{l}\text { Documentos sobre } \\
\text { análisis del } \\
\text { comportamiento del gasto } \\
\text { público agropecuario } \\
\text { costarricense, emitidos } \\
\text { por SEPSA. }\end{array}$ \\
\hline Organización & $\begin{array}{l}\text { Personas e instituciones } \\
\text { formales responsables de } \\
\text { una política o que inciden } \\
\text { en ella }\end{array}$ & $\begin{array}{l}\text { Comunicaciones } \\
\text { personales con } \\
\text { funcionarios públicos del } \\
\text { sector en estudio }\end{array}$ \\
\hline
\end{tabular}

Elaboración propia con base en Fontaine $(2015,162)$.

La incorporación al análisis de estas categorías se sustenta en dos supuestos básicos. En primer lugar, es importante tener claro que los desafíos actuales del desarrollo rural son multidimensionales y se encuentran interconectados a otra serie de problemáticas que trascienden las fronteras del mundo rural, o eminentemente agrícola. La compleja relación entre las dinámicas rurales y los retos estructurales del desarrollo territorial nos obliga a repensar y ser más críticos de los marcos de interpretación que, de forma tradicional, han dado forma al imaginario político sobre la ruralidad y a las diferentes narrativas del desarrollo que parecen tener asidero en la institucionalidad pública.

De esta manera, la revisión de los instrumentos que establecen lineamientos específicos para el desarrollo rural debe realizarse de tal modo que permita esclarecer el andamiaje institucional que se encuentra detrás del diseño y la 
formulación de políticas de desarrollo. Un ejercicio de tal naturaleza contribuye a dilucidar preguntas clave sobre la racionalidad de las políticas de desarrollo rural y su componente normativo; así como otros elementos muy influyentes en el diseño y la formulación de los distintos instrumentos; por ejemplo: el rol de los grupos de poder en diferentes escalas (local, regional, nacional), la legitimidad de las instituciones públicas para atender demandas territorialmente localizadas y la pertinencia del acompañamiento público en la identificación de opciones de desarrollo que vienen gestándose desde ámbitos externos al mandato público-gubernamental.

Por dichos motivos, al colocar en perspectiva analítica las categorías indicadas en el cuadro 1, pretendemos lograr una comprensión más detallada de la articulación entre la racionalidad política que precede al diseño de los instrumentos estudiados y las problemáticas persistentes de desarrollo. Su atención podría requerir de una mejor articulación interinstitucional o del liderazgo colaborativo de diferentes actores con capacidades complementarias.

En segundo lugar, la incorporación de los elementos de la sociología de la acción pública enriquece el enfoque del desarrollo territorial porque permite, por una parte, generar una mejor reflexión sobre el proceso de construcción de toma de decisiones y la calidad de los flujos de información que circulan desde lo institucional a lo local y viceversa. Las categorías de autoridad y tesoro, por otra parte, brindan un panorama más claro sobre los efectos del debilitamiento estatal y la disminución de sus capacidades estructurales para atender amenazas crecientes y nuevas. Evidentemente, estos aspectos guardan relación directa con la preparación del Estado para planificar un desarrollo rural acorde con el contexto de intervención.

Uno de los principios básicos que se encuentra detrás de tal idea es, justamente, problematizar la incorporación del análisis sistémico y la perspectiva multiactor en la identificación de soluciones potenciales a los principales problemas rurales. Podría, además, contribuir al establecimiento de sinergias y alianzas que disminuirían los costos de transacción que tendría implementar determinadas políticas, y de esta forma lograr una mejor articulación entre las políticas públicas y el desarrollo territorial rural.

No obstante, la experiencia latinoamericana nos demuestra que existe una evidente fragmentación institucional en el abordaje de las problemáticas rurales: muchas veces en función de las agendas y los compromisos de las carteras ministeriales, y que dejan sin resolver las causas subyacentes que amenazan con el boicot de cualquier iniciativa con algún grado de éxito.

Esta compartimentalización de las instituciones públicas conduce a que la intervención de problemáticas complejas también se lleve a cabo de forma fragmentada, lo cual se convierte en un obstáculo para entender la interacción entre agendas de desarrollo complementarias; por ejemplo: género y ruralidad, juventud y conservación del ambiente, por citar solo dos casos.

Adicionalmente, tenemos claro que existe una relación directa entre el desarrollo y la desigualdad en América Latina. Un hecho innegable es que la desigualdad en varios de los países de la región, en particular la desigualdad de 
ingreso, aumenta incluso en los lapsos de crecimiento económico, lo cual representa un desafío evidente para las políticas públicas (Dutrénit y Sutz 2014, 8).

A nivel territorial, estas problemáticas tienen efectos diferenciados. El desarrollo desigual entre territorios urbanos y rurales continúa siendo una de las principales preocupaciones para los gobiernos locales y nacionales, y también para los formuladores de política pública (Samper y Torrens 2015, 16). La desigualdad territorial es un fenómeno multicausal y altamente complejo, asociado con frecuencia con la alta concentración espacial de la población en áreas urbanas que, por lo general, presentan crecimiento económico pero escasa planificación; y áreas rurales que pese a aumentar en número de habitantes, mantienen opciones productivas y oportunidades de desarrollo limitadas. (CEPAL 2010, 228),

En Costa Rica, la persistencia de las asimetrías territoriales ha derivado en la aparición de polos de desarrollo en algunos centros urbanos, en detrimento del aumento de la exclusión en los territorios rurales. El más reciente informe del Estado de la Nación, publicado en el 2018, concluye que la persistencia de la desigualdad urbano-rural es un tema preocupante, al afirmar que en Costa Rica la pobreza afecta más a los hogares de zonas rurales, los cuales perciben ingresos que $41,7 \%$ inferiores en comparación con los hogares urbanos. Asimismo, la calificación de los trabajadores en zonas rurales es más baja, al igual que las oportunidades de empleo, principalmente entre mujeres rurales. Adicionalmente, la inequidad de género se agrava por las limitadas fuentes de trabajo en los territorios rurales (PEN-CONARE 2018, 49).

La institucionalidad del sector agropecuario y rural en Costa Rica también ha experimentado cambios importantes, entre ellos, la transformación del Instituto de Desarrollo Agrario (IDA) en el Instituto de Desarrollo Rural (INDER), en el 2012. En este sentido, el INDER se ha propuesto consolidar una nueva ruta institucional y sectorial, con el fin de impulsar una agenda de desarrollo rural basado en un esquema planificado y metodologías de trabajo colaborativo con los actores locales.

Este cambio institucional parte de la tesis de que es necesaria una nueva visión para enfrentar los múltiples desafíos que se presentan en los territorios rurales de Costa Rica, desde un enfoque territorial del desarrollo rural y de cambio gradual en las políticas públicas. Así, el desarrollo territorial se definió en la Ley n. ${ }^{\circ} 9036$ como un proceso multidimensional, multisectorial, que reconoce la diversidad de las economías territoriales, que trasciende los aspectos netamente agroproductivos:

Un proceso de cambio integral en materia económica, social, cultural e institucional, impulsado en un territorio rural, con la participación concertada y organizada de todos los actores sociales existentes en dicho territorio, orientado a la búsqueda de la competitividad, la equidad, el bienestar y la cohesión e identidad social de sus pobladores (INDER 2012, p. 2). 
En términos de la importancia del enfoque para el desarrollo de los territorios rurales y, de manera específica, para el diseño de políticas dirigidas a este propósito, Favareto (2007) señala que este enfoque contribuye a:

- Avanzar en la identificación de iniciativas de transformación productiva que responden de manera asertiva a las especificidades y problemas de un territorio o región en concreto.

- Movilizar actores sociales a través de formas novedosas de coordinación multinivel y el diseño de agendas intersectoriales de desarrollo.

- Facilitar espacios de coordinación y articulación, en torno a una visión de futuro compartida por las partes interesadas.

Dicho enfoque surgió primero en Europa, en los años noventa, y tiene su expresión más clara en los programas LEADER (FAO 2007, 18). Sin embargo, no fue hasta inicios del actual siglo que comenzó a implementarse en los países latinoamericanos, promovido principalmente por las organizaciones internacionales y regionales con presencia en el área, entre ellos, el Banco Interamericano de Desarrollo (BID), el Instituto Interamericano de Cooperación para la Agricultura (IICA) y el Fondo Internacional de Desarrollo Agrícola (FIDA).

Propone una visión alternativa para abordar los problemas estructurales de los territorios rurales (Schejtman y Berdegué 2003, 13; Samper y Torrens $2015,9)$ y plantea una visión del desarrollo rural que se distancia de las perspectivas tradicionales, basadas en las dinámicas sectorial-productiva y de extensión rural. El enfoque territorial rural toma en cuenta las nuevas condiciones de la ruralidad, la multidimensionalidad de los territorios y la interacción con los actores sociales como elementos importantes de desarrollo, bienestar y crecimiento económico (Fernández 2016, 6).

En el marco de tal concepción, el territorio adquiere un nuevo protagonismo como variable de análisis en el estudio de las causas y factores que explican por qué algunos territorios logran desarrollarse ambiental, social y económicamente y por qué otros se quedan estancados en ese camino (Berdegué, Carriazo, Jara, Modrego y Soloaga 2015, 59). En la figura 1, se establecen los principales componentes que enriquecen el enfoque de desarrollo de territorios rurales.

Figura 1. Componentes del enfoque de desarrollo territorial rural

\begin{tabular}{l|l}
\hline Transformación productiva & Desarrollo Institucional \\
\hline Tiene el propósito de articular & Tiene como objetivo estimular la concertación \\
competitiva y sustentablemente a & de los actores locales entre sí y entre ellos y \\
la economía del territorio con & los agentes externos relevantes, así como \\
mercados dinámicos, lo que & modificar las reglas formales e informales que \\
supone cambios en los patrones & reproducen la exclusión de los pobres en los \\
de empleo y producción de un & procesos y los beneficios de la transformación \\
espacio rural determinado. & productiva. \\
\hline
\end{tabular}

Elaboración propia, con base en Schejtman y Berdegué $(2003,24)$. 
1. Comunicación personal con Harys Regidor Barboza.

Presidente ejecutivo del Instituto de Desarrollo Rural (INDER). 18 de enero de 2018 Instalaciones del INDER. San José, Costa Rica.
Ambrosio-Albalá y Bastiaensen (2010) plantean que el enfoque territorial del desarrollo rural permite la implementación de estrategias de política enfocadas en la participación de los actores locales, gracias a la articulación entre los diferentes sectores y niveles de gobernanza territorial. El objetivo principal es mejorar la formulación e implementación de una nueva generación de políticas para combatir la pobreza rural desde una perspectiva intersectorial.

En Costa Rica, la aplicación de dicho enfoque es aún incipiente. No obstante, existe un reconocimiento claro por parte de las instituciones públicas, en especial el INDER, de la importancia de su incorporación al diseño de políticas territoriales con visión integral, particularmente aquellas cuya finalidad es reactivar y acelerar el desarrollo en los territorios rurales, mediante la activación productiva y competitiva, y el fortalecimiento del capital social ${ }^{1}$.

\section{Resultados: evolución de las políticas e instrumentos para el desarrollo rural en costa rica a partir del 2015}

\section{Evolución y cambios recientes en el sector agropecuario de Costa Rica}

De acuerdo con Saénz Segura y Chaves Moreira (2014), la evolución de la institucionalidad agropecuaria puede caracterizarse a partir de tres etapas o momentos históricos precisos. Anterior a 1948, es posible identificar un periodo liberal que coincide con la creación de las primeras oficinas de agricultura, en una Costa Rica con escasa población y retos importantes en materia de desarrollo agroproductivo. Durante esta etapa, una de las principales metas de la incipiente política agropecuaria del país es asegurar una agricultura de abastecimiento de alimentos, mediante la promoción de actividades tradicionales como la producción de café, banano, caña de azúcar y ganadería.

En 1949-1950, con las reformas de la Junta Fundadora de la Segunda República, emergió una visión estatista del desarrollo, mediante la creación de empresas públicas para impulsar la economía y colonizar el país. Este hecho se materializó con una expansión significativa de la frontera agrícola, en las décadas de 1950 y 1960, y la creación de tres organizaciones clave para el sector. En 1949, por medio de la nueva Constitución Política, se creó el Ministerio de Agricultura e Industria, que en 1960 por medio de la Ley de presupuesto n. ${ }^{\circ}$ 2656, adoptaría el nombre de Ministerio de Agricultura y Ganadería (MAG), una institución rectora de la política agraria del país e impulsora de la investigación agropecuaria, fitotécnica y zootécnica. El Instituto Costarricense de Tierras y Colonización (ITCO) se creó bajo la Ley n. ${ }^{\circ} 2825$, del 14 de octubre de 1961, denominada por su naturaleza Ley de Tierras y Colonización, como un medio de formalizar lo que en la práctica estaba sucediendo con los llamados "denuncios", convirtiéndolos en procesos de colonización agrícola. EI ITCO es considerado el predecesor del IDA. En 1956, mediante la Ley 2035, se crea el Consejo Nacional de la Producción, cuya función es la co- 
mercialización de la producción para abastecer los mercados locales y regionales.

En 1980, la incorporación de los Programas de Ajuste Estructural (PAE) contribuyó en gran medida a que modelo vigente hasta entonces entre en crisis, al producirse un replanteamiento significativa de la función del Estado en la actividad agropecuaria, que conduciría a una mayor liberalización de los mercados. En fechas más recientes, la institucionalidad agropecuaria ha resultado afectada en diferente medida con el fortalecimiento de los marcos institucionales que promueven la protección del ambiente, basada en una mayor regulación de las actividades agroproductivas. Las iniciativas de reforestación y reverdecimiento de la economía se mantienen vigentes hasta nuevos días (Sáenz-Segura, Le Coq y Bonin 2017).

Posterior al 2000, las instituciones del sector agropecuario, incluyendo las 11 organizaciones públicas, corporaciones, cámaras y otros actores privados, se han encargado de promover nuevas agendas temáticas, asociadas principalmente al cambio tecnológico y la modernización del campo, vía transferencia de tecnologías e instrumentos para asegurar la integración de encadenamientos productivos locales a las cadenas globales de valor.

No obstante, para Barboza-Arias y Saénz-Segura (2019), también es fundamental mencionar la prevalencia de retos del sector, como los problemas de fragmentación institucional y la falta de mecanismos efectivos para la coordinación y el trabajo articulado, con el fin de atender de manera integral los temas trasversales como la seguridad alimentaria, la gobernanza del recurso hídrico y la creación de mercados locales.

Con respecto a la evolución y los cambios ocurridos en la forma de abordar la cuestión territorial, cabe resaltar que el cambio de IDA a INDER representa una transición crucial, no solo en términos de la racionalidad de los esquemas, sino también en la intencionalidad del enfoque. La función primordial del IDA, al estar focalizada en la creación y el desarrollo de los asentamientos campesinos, suele asociarse con la regularización de los procesos de toma de tierras y la incorporación de procesos técnicos de titulación, delimitación de tierras y entrega de propiedades, en un contexto histórico marcado por las guerras civiles en América Central (durante la década de los años ochenta) y la amenaza de la infiltración del conflicto en territorial nacional. Por su parte, el INDER se constituye sobre una lógica de desarrollo rural, que incorpora y transciende el énfasis en los asentamientos campesinos. Así pues, se orienta a la consolidación de servicios públicos bajo un enfoque de región y de promoción de actividades con mayor cobertura territorial.

\section{Periodo 2015-18. Administración Solís Rivera (2014-2018)}

En el Plan Nacional de Desarrollo 2015-2018 se menciona la necesidad de promover un proceso efectivo de concertación sectorial e intersectorial, en especial con el Ministerio de Planificación Nacional y Política Económica (MIDEPLAN), que evite la duplicidad de funciones y les permita a las instituciones desarrollar acciones en los territorios rurales de forma organizada. 
2. A marzo de 2019, existían 29 territorios rurales plenamente constituidos.

3. Se planteó una reducción de la pobreza rural en 6.5 puntos porcentuales. Cada uno de estos objetivos se subdividió en programas o proyectos específicos.
Se reconoce que en el país no existen las condiciones institucionales para que los organismos estatales presenten proyecto de intervención integrada en la totalidad de los territorios rurales ${ }^{2}$, cuya delimitación y clasificación se encuentra a cargo del INDER.

El capítulo agropecuario que se incorporó en el PND 2015-18 y el Plan Sectorial de Desarrollo Agropecuario representan los dos instrumentos principales por los cuales se implementaron las políticas agropecuarias y rurales durante la administración Solís Rivera, de acuerdo a dos objetivos estratégicos sectoriales: 1. Aumentar el valor agregado agropecuario, con un crecimiento entre 6 y $8 \%$ en el periodo, y, 2. Apoyar la meta nacional de reducción de la pobreza en los territorios rurales y propiciar la dignificación de la población rural $^{3}$.

\section{Cuadro 2. Acciones de política e institucionales para el Desarrollo Rural} Territorial. 2015-2018

\begin{tabular}{|c|c|c|}
\hline $\begin{array}{c}\text { Área } \\
\text { estratégica }\end{array}$ & $\begin{array}{c}\text { Acciones estratégicas de } \\
\text { política }\end{array}$ & $\begin{array}{l}\text { Acciones estratégicas } \\
\text { institucionales }\end{array}$ \\
\hline \multirow{8}{*}{$\begin{array}{l}\text { Encadenamiento } \\
\text { s productivos y } \\
\text { cadenas de } \\
\text { valor basados } \\
\text { en las } \\
\text { potencialidades } \\
\text { de los territorios }\end{array}$} & \multirow{6}{*}{$\begin{array}{l}\text { Formulación y ejecución de } \\
\text { proyectos productivos con valor } \\
\text { agregado, con apoyo de fondos } \\
\text { de riesgo u otros mecanismos de } \\
\text { financiamiento }\end{array}$} & $\begin{array}{l}\text { Promover la dotación de la } \\
\text { tecnología, capital, trabajo y acceso } \\
\text { a mercados }\end{array}$ \\
\hline & & $\begin{array}{l}\text { Adquisición de tierras para la } \\
\text { implementación de proyectos } \\
\text { productivos }\end{array}$ \\
\hline & & $\begin{array}{l}\text { Inversión en proyectos de } \\
\text { infraestructura rural }\end{array}$ \\
\hline & & $\begin{array}{l}\text { Inversión en proyectos productivos } \\
\text { mediante el otorgamiento de } \\
\text { créditos }\end{array}$ \\
\hline & & $\begin{array}{l}\text { Promoción de las organizaciones de } \\
\text { productores(as) y juventud rural }\end{array}$ \\
\hline & & $\begin{array}{l}\text { Aumento en el acceso de servicios } \\
\text { de organizaciones de productores }\end{array}$ \\
\hline & \multirow{2}{*}{$\begin{array}{l}\text { Integración de las comunidades } \\
\text { en las Áreas Marinas de Pesca } \\
\text { Responsable (AMPR) para la } \\
\text { conformación de centros } \\
\text { regionales pesqueros y acuícolas }\end{array}$} & $\begin{array}{l}\text { Implementar el Sello de } \\
\text { Denominación de Origen en AMPR }\end{array}$ \\
\hline & & $\begin{array}{l}\text { Fortalecimiento de las comunidades } \\
\text { costeras y acuícolas }\end{array}$ \\
\hline $\begin{array}{l}\text { Desarrollo } \\
\text { Humano e } \\
\text { inclusión social } \\
\text { en los territorios }\end{array}$ & $\begin{array}{l}\text { Coordinación y articulación de } \\
\text { esfuerzos con las instituciones } \\
\text { públicas y privadas }\end{array}$ & $\begin{array}{l}\text { Transferencia de recursos } \\
\text { presupuestarios para la ejecución } \\
\text { de proyectos de desarrollo rural }\end{array}$ \\
\hline \multirow{2}{*}{$\begin{array}{l}\text { Creación de las } \\
\text { instancias de } \\
\text { convergencia } \\
\text { público-privado } \\
\text { de desarrollo en } \\
\text { los territorios }\end{array}$} & \multirow[b]{2}{*}{$\begin{array}{l}\text { Instauración de los consejos } \\
\text { territoriales y elaboración de los } \\
\text { planes territoriales }\end{array}$} & Conformación de los CTDR* \\
\hline & & $\begin{array}{l}\text { Formulación de los planes } \\
\text { territoriales de desarrollo }\end{array}$ \\
\hline \multicolumn{3}{|c|}{$\begin{array}{l}\text { * Los consejos territoriales y los consejos regionales de desarrollo rural agrupan los actores tanto } \\
\text { públicos como privados en los territorios y regiones. Estos actores deben estar armonizados con los } \\
\text { planes reguladores municipales y otras acciones del sector público de acuerdo con los objetivos } \\
\text { presentes en la Ley n. }{ }^{\circ} 9036 \text {. Desde tales espacios, se formulan los planes. }\end{array}$} \\
\hline
\end{tabular}

(SEPSA, 2015, 12). 
La Secretaría Ejecutiva de Planificación Sectorial Agropecuaria (SEPSA), de acuerdo a la Ley Fodea n. ${ }^{\circ} 7064$, estableció acciones complementarias específicas para la ejecución efectiva del plan sectorial. Algunas de estas acciones representan la formalización de programas o proyectos que derivan directamente de los objetivos estratégicos sectoriales del PND; tales son los casos del Plan Nacional de Seguridad Alimentaria y Nutricional y el Plan Sectorial de Género y Juventud Rural.

Se definieron cinco pilares de acción, orientados al cumplimiento de dichos objetivos. Uno de ellos es justamente el desarrollo rural territorial. La principal área estratégica en la que se concentró dicho pilar fue la promoción de encadenamientos productivos y cadenas de valor; pero también se consideran el desarrollo humano con inclusión social y la convergencia público-privado como áreas estratégicas importantes (Cuadro 2).

Periodo 2019-2022. Plan nacional de desarrollo y de inversión pública del bicentenario. Administración Alvarado Quesada (20182022)

Uno de los principales avances en el PND de la administración Alvarado Quesada (2018-2022) es la incorporación de un capítulo sobre desarrollo territorial, que propone la regionalización como uno de los ejes centrales en la planificación institucional del país. De esta manera, se da continuidad a lo establecido en el Decreto Ejecutivo 16068-, Plan de Reforma División Regional del Territorio de Costa Rica.

Otros aspectos relevantes son el diagnóstico de potencialidad productiva por territorio que se introduce en el marco de la propuesta de regionalización. Se identifican las principales particularidades propias de zonas rurales, fronterizas y costeras, así como las grandes potencialidades de recursos naturales y humanos que pueden generar desarrollo endógeno e insertar la economía local en los mercados externos. Según se indica en el Plan Nacional de Desarrollo y de Inversión Pública del Bicentenario, de la administración Alvarado Quesada (2018-2022), la meta principal del desarrollo territorial es aumentar la ocupación laboral dentro de las regiones periféricas, mediante un modelo de gestión participativo que promueva el desarrollo (MIDEPLAN 2019, 21).

Con respecto a las políticas agropecuarias, se dará continuidad a los esfuerzos para aumentar el valor agregado agropecuario, la transferencia tecnológica a las Pymes y la generación de productos diferenciados, gracias de la innovación, temas que habían sido una prioridad en la administración Solís Rivera (2014-2018). En el cuadro 3, se presentan de forma esquemática los principales programas y objetivos que se derivan de estos lineamientos del PND. 
Cuadro 3. Intervenciones estratégicas y objetivos en el sector agropecuario y rural (2018-2022)

\begin{tabular}{|l|l|}
\hline \multicolumn{1}{|c|}{ Intervención estratégica } & \multicolumn{1}{c|}{ Objetivo } \\
\hline Programa de Producción Sostenible & $\begin{array}{l}\text { Desarrollar modelos de producción } \\
\text { sostenibles en fincas ganaderas y } \\
\text { agrícolas }\end{array}$ \\
\hline $\begin{array}{l}\text { Programa Nacional de Protección del } \\
\text { Patrimonio Agropecuario Nacional y la } \\
\text { salud pública }\end{array}$ & $\begin{array}{l}\text { Aumentar el control y vigilancia zoo y } \\
\text { fitosanitaria }\end{array}$ \\
\hline $\begin{array}{l}\text { Programa Nacional de Control Oficial } \\
\text { de Calidad de }\end{array}$ & $\begin{array}{l}\text { Controlar el cumplimiento de normas } \\
\text { oficiales de calidad de semilla } \\
\text { demandada en el mercado }\end{array}$ \\
\hline $\begin{array}{l}\text { Programa de inserción efectiva de los } \\
\text { jóvenes egresados de los liceos rurales } \\
\text { y colegios }\end{array}$ & $\begin{array}{l}\text { Generar emprendimientos productivos } \\
\text { con jóvenes egresados de los liceos } \\
\text { rurales y colegios técnicos }\end{array}$ \\
\hline $\begin{array}{l}\text { Proyecto Sistema Control de } \\
\text { Inundaciones Área del río Limoncito, } \\
\text { Limón }\end{array}$ & $\begin{array}{l}\text { Reducir los riesgos de inundación en } \\
\text { zonas vulnerables }\end{array}$ \\
\hline $\begin{array}{l}\text { Programa de Abastecimiento } \\
\text { Institucional (PAI) }\end{array}$ & $\begin{array}{l}\text { Incrementar la participación de } \\
\text { agroempresas y organizaciones de la } \\
\text { economía social }\end{array}$ \\
\hline $\begin{array}{l}\text { Programa Nacional de Pesquerías } \\
\text { Sustentables de Atún y Grandes } \\
\text { Pelágicos }\end{array}$ & $\begin{array}{l}\text { Fomentar las pesquerías sustentables } \\
\text { de atún y grandes pelágicos }\end{array}$ \\
\hline $\begin{array}{l}\text { Elaboración propia con base en Plan Nacional de Desarrollo y de Inversión Pública del } \\
\text { Bicentenario (MIDEPLAN 2019, 16). }\end{array}$
\end{tabular}

\section{Evolución de la inversión y el gasto público agropecuario y rural}

El Programa sectorial de desarrollo rural territorial representó $12,6 \%$ del gasto público agropecuario ejecutado en 2017 , lo cual corresponde a un monto de 24 332, 1 millones de colones costarricenses (40 millones USD, aproximadamente). Estos recursos se destinaron, en su mayoría, a la gestión agraria y regulación de la tierra y la gestión para el desarrollo territorial (SEPSA 2018, 4).

Del total de recursos ejecutados en el programa, 36,7\% (alrededor de 8 921,2 millones de colones costarricenses) se destinaron a proyectos específicos en territorios, relacionados con infraestructura, seguridad alimentaria y crédito rural. En conjunto, la inversión en esos proyectos benefició a cerca 28 451 hogares.

Estas intervenciones presentan como preocupaciones transversales la pobreza y el desempleo rurales, y buscan combatir la migración rural-urbana (MIDEPLAN 2014, 17). A nivel económico, la promoción de encadenamientos productivos mediante el impulso a la infraestructura y el crédito rural buscan contrarrestar la reducción del área destinada al cultivo de los granos básicos y la creciente dependencia a las importaciones de esos productos. Con res- 
pecto al presupuesto aprobado y ejecutado para las principales instituciones del sector agropecuario y rural, la información se presenta en el cuadro 4.

Cuadro 4. Costa Rica: Presupuesto agropecuario en millones de colones: montos aprobados y ejecutados según institución (2014-2017)

\begin{tabular}{l|r|r|r|r|r|r|r|r}
\hline $\begin{array}{c}\text { Institu } \\
\text { ción }\end{array}$ & \multicolumn{2}{|c|}{2014} & \multicolumn{2}{c|}{2015} & \multicolumn{2}{c|}{2016} & \multicolumn{2}{c}{2017} \\
\hline & $\begin{array}{c}\text { Monto } \\
\text { aprobado }\end{array}$ & $\begin{array}{c}\text { Monto } \\
\text { ejecutado }\end{array}$ & $\begin{array}{c}\text { Monto } \\
\text { aprobado }\end{array}$ & $\begin{array}{c}\text { Monto } \\
\text { ejecutado }\end{array}$ & $\begin{array}{c}\text { Monto } \\
\text { aprobado }\end{array}$ & $\begin{array}{c}\text { Monto } \\
\text { ejecutado }\end{array}$ & $\begin{array}{c}\text { Monto } \\
\text { aprobado }\end{array}$ & $\begin{array}{c}\text { Monto } \\
\text { ejecutado }\end{array}$ \\
\hline INDER & 37806,9 & 26499,9 & 45037,5 & 35522,0 & 48069,9 & 39233,2 & 51365,4 & 34865,4 \\
\hline MAG & 48432,6 & 47454,3 & 54893,7 & 52665,4 & 47083,8 & 45303,9 & 41213,5 & 42583,4 \\
\hline INTA & 1936,5 & 1348,5 & 2058,9 & 1260,8 & 1991,3 & 1393,0 & 1224,5 & 1167,6 \\
\hline
\end{tabular}

Elaboración propia con base en SEPSA 2017, 5.

Como se observa, a partir del 2016 el presupuesto aprobado para el INDER ha sido superior al otorgado al MAG, incluso cuando este ministerio debe asumir programas que requieren una inversión importante, entre ellos, la Dirección Nacional de Extensión Agropecuaria (DNEA). Por otro lado, se evidencia también una tendencia decreciente en el presupuesto aprobado para estas instituciones, una situación que posiblemente se mantuvo en 2018.

En el caso de INDER, en 2017 la institución tuvo un porcentaje de ejecución presupuestaria inferior a $80 \%$. Esto se debe a la baja ejecución del programa 1: Actividades Centrales, en las partidas de servicios; por ejemplo, no se pudo finalizar las consultorías programadas, así como otros servicios de gestión y apoyo, capacitación y desarrollo de sistemas. Otras subejecuciones se presentaron en partidas de materiales y suministros, bienes duraderos, y transferencias corrientes (SEPSA, 2017).

\section{Discusión}

Históricamente, la pobreza no extrema rural (PNER) ha superado a la pobreza no extrema urbana (PNEU). En el caso de la pobreza extrema rural (PER), esta incluso duplicó a la pobreza extrema en las zonas urbanas (PEU) durante periodos específicos (ver gráfifco 1).

Si bien la PNER disminuyó en 2015 y 2016, en 2018 volvió a ubicarse en $16,8 \%$ (gráfico 1), lo cual representa 235775 hogares rurales en esa condición. Por otra parte, aunque la PER logró reducir en casi dos puntos porcentuales en el lapso 2016-2017, en 2018 volvió a incrementarse. Significa que la meta de reducción de la pobreza en 6.5 puntos porcentuales de la administración Solís Rivera no se cumplió. Si analizamos la pobreza por regiones de planificación, mientras que en el 2018, la pobreza extrema en la región Central, considerada la zona más urbana del país, era de $4,4 \%$, en zonas rurales 
como la Región Brunca y la Huetar Norte fue de 10,1 \% y 10,0 \%, respectivamente.

\section{Gráfico 1. Costa Rica: Distribución relativa de los hogares por nivel de pobreza, según año, julio 2012 - 2018}

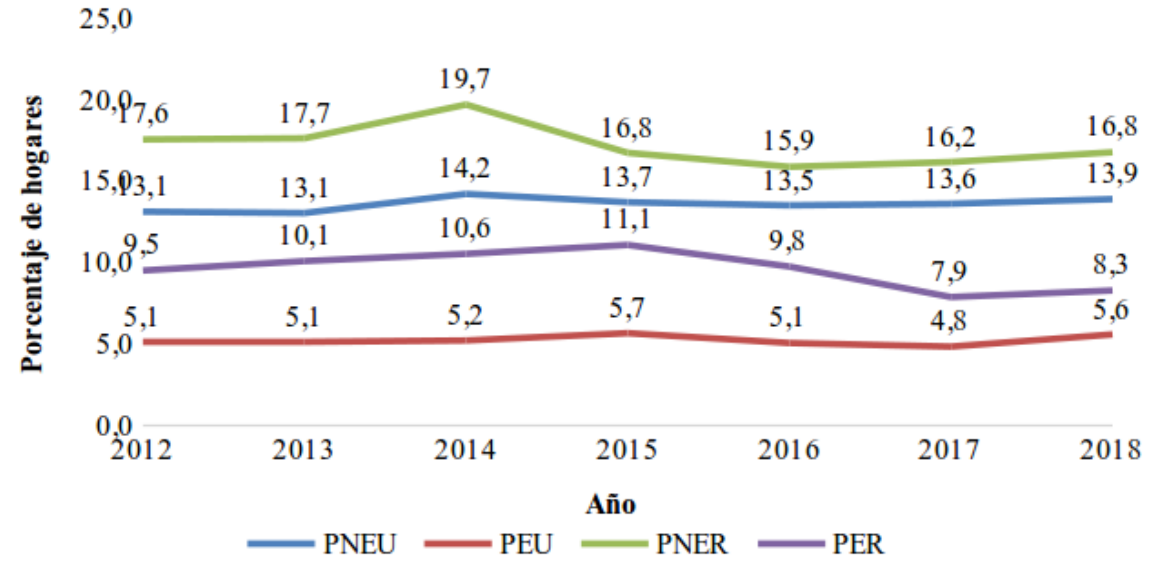

Elaboración propia con base en ENAHO (INEC 2019).

Con respecto al desempleo rural, en 2018 la población rural desempleada representó cerca del $10 \%$ de la fuerza de trabajo rural. Como se observa en el gráfico 2, si se considera el grupo etario, la población rural más afectada por el desempleo siguen siendo los jóvenes.

Gráfico 2. Costa Rica: Población rural desempleada por edad, según trimestre,

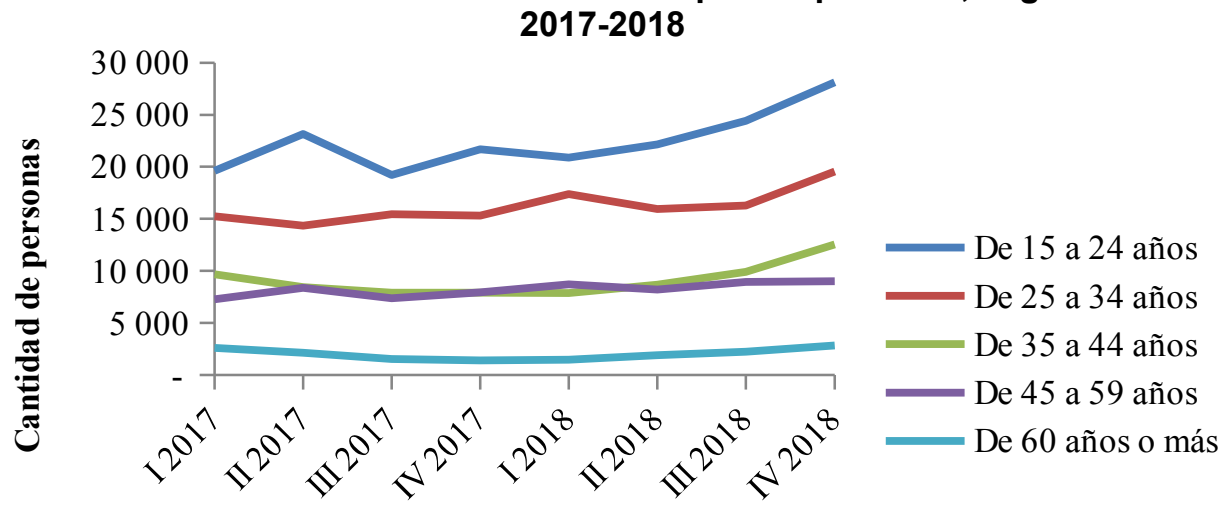

Período

Elaboración propia con base en ENAHO (INEC 2019). 
El desempleo rural afecta principalmente a personas con grado de secundaria o una calificación inferior (gráfico 3).

\section{Gráfico 3. Población rural desempleada por calificación, según trimestre,} 2017-2018

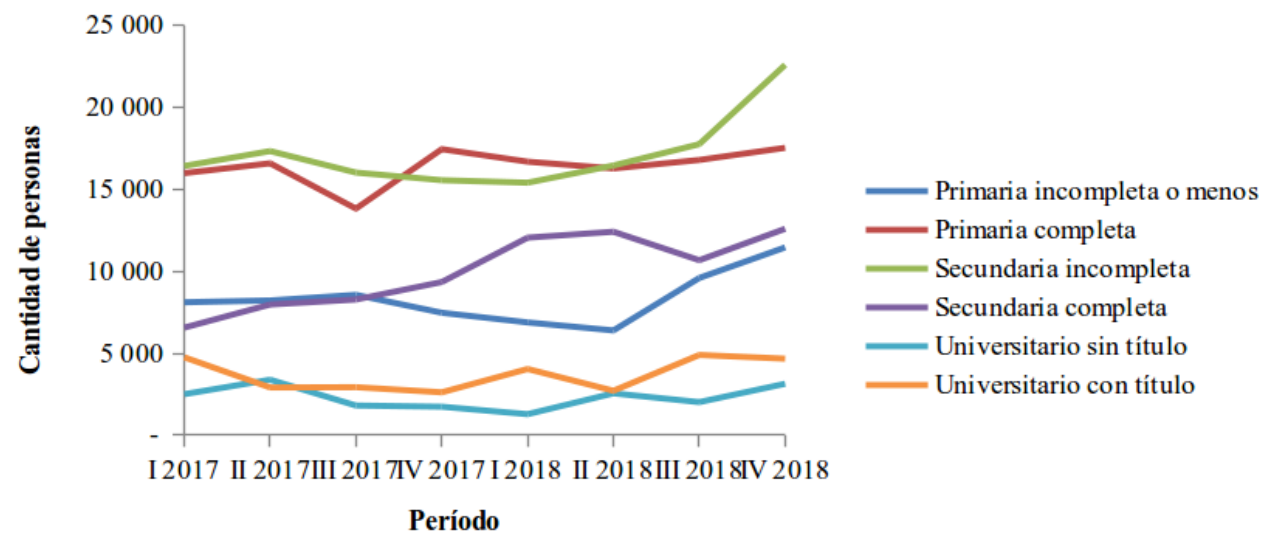

Elaboración propia con base en ENAHO (INEC 2019).

Pese a que Costa Rica aprobó en el 2010 la Estrategia Centroamericana de Desarrollo Rural Territorial (ECADERT), en el marco del Sistema de Integración Centroamericana (SICA), con el propósito de generar nuevas oportunidades y fortalecer las capacidades de la población de los territorios rurales (ECADERT 2010, 77), los datos anteriores evidencian que esa y otras políticas implementadas no han obtenido los resultados que se proponían. Una de las principales razones por las que ocurre esta situación se debe a que estos instrumentos no logran reflejar las dinámicas particulares del empleo rural y la manera en que los jóvenes menos calificados se insertan en las actividades productivas.

Mediante la especialización en tareas mecánicas, que pueden resultar básicas en términos del uso intensivo de conocimiento o tecnología, muchos jóvenes desarrollan una curva de aprendizaje que luego les resulta difícil, incluso imposible, de replicar en otros sectores. Por lo tanto, cuando las políticas y lineamientos del empleo rural y la inclusión de la juventud al mercado laboral se basan en desplazarlos hacia sectores productivos que son considerados más competitivos o que presentan mejor desempeño económico, no se está comprendiendo bien el trasfondo de las capacidades adquiridas como resultado de la experiencia cotidiana y el conocimiento tácito.

De igual forma, se pierde de vista con relativa facilidad que no todos los jóvenes rurales reúnen aptitudes para el emprendedurismo en igual medida. Si bien para algunos ésta puede ser la estrategia correcta, en otros casos es 
importante una estrategia diferente que potencie otro tipo de habilidades y capacidades en los jóvenes y la población rural desempleada. Lo anterior incluye la creación de nuevos mercados, un tema que actualmente no se discute de forma explícita.

De acuerdo con los Planes Nacionales de Desarrollo, la pobreza y el desempleo son las principales problemáticas del desarrollo rural en Costa Rica. Los programas y otros instrumentos analizados se orientan a atender ambos fenómenos de modo transversal. Juventud y mujer son los rostros humanos de estas iniciativas públicas. No obstante, la eficiencia y sostenibilidad de las acciones propuestas pueden estar comprometidas en el mediano y largo plazo, y no alcanzar los resultados esperados. La generación de empleo, por ejemplo, debe promoverse en función de una política de desarrollo integral, y no solamente desde la política sectorial agroproductiva, de diversificación y generación de valor agregado, por medio de la cual se privilegian actividades enfocadas en los problemas de producción solamente.

En la misma línea, las políticas deben ser reflexivas para lograr una articulación efectiva con las dinámicas socio-históricas, culturales y ambientales de los territorios. El componente multinivel al que refieren ambos PND amerita un mayor contenido estratégico; para ello, es indispensable repensar los esquemas de articulación interinstitucional vigentes, con el objetivo de lograr una mejor gobernanza en la ejecución de las acciones. Se requieren arreglos institucionales que garanticen más compromiso político con respecto a la determinación de liderazgos y la promoción de vínculos cooperativos que resulten legítimos y significativos para los actores locales.

Otro aspecto por considerar es que una mayor presencia institucional en los territorios rurales no garantiza que la agenda de desarrollo territorial esté avanzando. La reproducción de la desigualdad es un tema estructural y, por ende, cualquier medida orientada a su tratamiento debe reconocer (de forma explícita) que existen relaciones de poder históricas que comprometen el alcance de las acciones más específicas.

No obstante, resulta alentador que se esté brindando una mayor atención a la importancia del enfoque de desarrollo territorial, con el objetivo de superar los obstáculos administrativos que obstaculizan la gestión territorial desde las organizaciones de base. El impulso que ambas administraciones han dado a los Consejos Territoriales de Desarrollo Rural (CTDR) ha permitido generar reflexiones en torno a la precarización laboral, la feminización de la pobreza, la expulsión de los jóvenes a la ciudad y el impacto del crimen organizado en los territorios rurales.

De igual manera, propuestas como la de regionalización, que promueve la actual administración Alvarado Quesada, han comenzado a problematizar las dimensiones geográficas, climatológicas y etnográficas de las cadenas de valor. Tal ejercicio resulta útil porque les permite comprender a los ejecutores de los instrumentos que algunas cadenas de valor que son exitosas en ciertos contextos no tendrían los mismos resultados si se trasladan a otros territo- 
rios, puesto que la configuración de fuerzas sociales y la interacción con el entorno natural son distintas.

Las políticas de homologación productiva en territorios rurales, que son utilizadas como estrategia de contención de la pobreza no contribuyen a los encadenamientos productivos locales porque constituyen un desfasamiento del sistema de producción local y de la organización social rural en sí misma.

\section{Conclusiones}

Como reflexión de cierre, desde el 2015 se observa un incremento importante del interés gubernamental en colocar el tema del desarrollo de los territorios rurales como uno de los ejes estratégicos de la agenda de desarrollo nacional. Existen esfuerzos notables para operacionalizar y dotar de contenido a los programas sectoriales que se encuentran asociados a objetivos e intervenciones para promover el desarrollo rural.

En esta misma línea, desde la administración Solís Rivera había tenido lugar una serie de arreglos institucionales que se orientan al establecimiento de acuerdos mínimos para alcanzar una coordinación entre actores, más eficiente y mejor articulada. Aunque de forma incipiente, se ha comenzado a debatir la necesidad de revisar las funciones que realizan las instituciones vinculadas, con el objetivo de mejorar las competencias y capacidades, y evitar así la duplicidad.

Empero, también vale la pena llamar la atención sobre la incorporación de elementos de gobernanza en la planificación del desarrollo rural. Es primordial continuar avanzando en el fortalecimiento del diálogo interinstitucional, y a la vez garantizar el apoyo formal a los liderazgos locales. En este contexto, las palabras clave son participación y empoderamiento.

Como ejercicio democrático, en la labor de las instituciones públicas debe operar un cambio de paradigma que contribuya a una mejor comprensión de las dinámicas territoriales. El diseño de las políticas debe avanzar hacia esquemas reflexivos, cuya racionalidad y contenidos puedan ser objeto de evaluación, crítica y redireccionamiento. Como apuntan Schejtman y Berdegué $(2003,35)$, la incorporación del análisis multinivel en la valoración de las necesidades y demandas de los actores locales facilita la promoción de acciones colectivas y el establecimiento de redes de trabajo con capital social dispuesto a asumir los retos más acuciantes del desarrollo. Por ende, las políticas públicas deben ser pensadas desde espacios de cogestión que contribuyan a potenciar las capacidades operativas de técnicos y beneficiarios.

En este sentido, la nueva ruta institucional en la que ha venido trabajando el INDER en los últimos años se enmarca en un proceso de transición y reorganización interna que tiene como propósito general movilizar a la sociedad civil y los actores locales hacia un escenario de transformación productiva inserto en la especificidad de cada territorio. Sin embargo, no se puede obviar que uno de los retos pendientes en esta materia es la incapacidad de las institu- 
ciones para abordar la noción de territorio rural como un sistema complejo. Esta situación se refleja en el análisis de los indicadores sobre pobreza y empleo rural, los cuales evidencian que la ausencia de un enfoque interseccional en la búsqueda y adopción de medidas integrales puede conducir incluso a más desigualdad, lo cual provocaría la vulnerabilización de grupos sociales específicos.

De igual manera, es imperativo que se amplíe la discusión pública en relación con las dinámicas de ejecución presupuestaria, que se orienta principalmente a gastos corrientes, y en que las partidas de remuneraciones y transferencias corrientes representan los porcentajes más altos. Como bien se reconoce en el análisis del comportamiento del gasto público agropecuario costarricense del 2017, esta distribución "no permite disponer de más recursos para invertir” (SEPSA 2018, p. 30). Tal situación afecta de manera significativa el desarrollo de acciones sustantivas, que bien podrían coadyuvar a revertir las brechas y rezagos entre los territorios rurales y urbanos.

Si a esto se suma el hecho de que la política agroproductiva del país resulta ineficiente para articular ejes de intervención estratégicos como mercado laboral, iniciativas de agregación de valor y competitividad territorial, un escenario plausible es que la reactivación económica de los territorios rurales pueda resultar comprometida en el futuro (Saénz Segura y Chaves Moreira, 2014),

Finalmente, para responder las preguntas planteadas en este estudio, se puede señalar que pese a estar presente de forma discursiva, vía referencia a acciones concretas para atender los retos de pobreza rural y activación económica, la incorporación del enfoque territorial a las políticas públicas de desarrollo rural no ha sido suficiente para generar los cambios institucionales que conducirían a una mejor planificación del desarrollo.

En lo que se refiere a las interrogantes específicas que se plantean, si bien existe un interés genuino en fortalecer los mecanismos territoriales de coordinación y cohesión social, la evidencia demuestra que espacios como los CTDR resultan escasos para acelerar la transformación productiva de los territorios rurales (Barboza-Arias y Saénz-Segura, 2019), desde una perspectiva multidimensional e intersectorial. Esto conlleva a que los efectos en el desarrollo sostenible se encuentren determinados en gran medida por las iniciativas de corto plazo, y en poca medida por verdaderas políticas de Estado, en capacidad de comprometer recursos y funciones en el largo plazo.

Al incorporar las categorías de la sociología de la acción pública al análisis, podemos concluir que las políticas de desarrollo rural han conservado un fuerte sesgo productivista, que dificulta el involucramiento de nuevos actores sociales en la construcción de procesos de toma de decisión a nivel local e institucional.

La ausencia de mecanismos institucionales que permitan articular los nuevos desafíos demográficos y del trabajo rural al diseño de políticas territoriales 
genera distorsiones significativas que originan, a su vez, más desigualdad y pobreza. Adicionalmente, la incapacidad de las administraciones gubernamentales para invertir en sectores estratégicos genera un desalineamiento entre las propuestas de reactivación económica y las condiciones históricas que han configurado la protección social en gran parte de los territorios rurales del país.

\section{Bibliografía}

Ambrosio-Albalá, M y Bastiaensen, J. (2010). The new territorial paradigm of rural development: Theoretical foundations from systems and institutional theories. Discussion Paper 2010.02. Institute of Development Policy and Management, University of Antwerp.

Barboza-Arias, L. M. y Saénz-Segura, F. (2019) Transición hacia un nuevo sistema de innovación agropecuaria en Costa Rica: evolución y retos. En Goulet, F. et al., (2019). Sistemas y políticas de innovación para el sector agropecuario en América Latina. Rio de Janeiro, Brasil: epapers.

Berdegué, Julio A., Fernando Carriazo, Benjamín Jara, Félix Modrego, and Isidro Soloaga. (2015). "Cities, territories, and inclusive growth: Unraveling urban-rural linkages in Chile, Colombia, and Mexico". World Development 73: 56-71.

CEPAL, NU. 2010. La hora de la igualdad: brechas por cerrar, caminos por abrir. Trigésimo Tercer Período de Sesiones de la CEPAL. Santiago, Chile: CEPAL.

Despacho de la Primera Dama de la República, Sra. Mercedes Peñas Domingo. 2015, marzo. Programa Tejiendo Desarrollo 2015-2018. Gobierno de la República de Costa Rica. Administración Solís Rivera.

Dutrénit, Gabriela, and Judith Sutz. 2014. Sistemas de innovación para un desarrollo inclusivo. La experiencia Latinoamericana. Editado por Gabriela Dutrénit y Judith Sutz. México DC: Foro Consultivo Científico y Tecnológico.

FAO. 2007. Desarrollo territorial rural. Análisis de experiencias en Brasil, Chile y México. Santiago, Chile: FAO.

FAO. 2018. Panorama de la pobreza rural en América Latina y el Caribe 2018. Santiago. Número de páginas (112). ISBN 978-92-5-131085-4, Licencia: CC BY-NC-SA 3.0 IGO.

Favareto, A. 2007. Paradigmas do desenvolvimento rural em questão. São Paulo: Iglu/Fapesp.

Fernández, Lisandro Federico. (2016). "El Desarrollo Territorial Rural y su influencia en las políticas para la agricultura familiar". 
Fontaine, Guillaume. 2015. El análisis de las políticas públicas. Conceptos, teorías y métodos. Barcelona, España: Anthropos.

IICA. 2010. Estrategia Centroamericana de Desarrollo Rural Territorial 20102030: ECADERT/ CAC, ECADERT, SICA. San José, C.R : IICA.

IMAS. 2015. Puente al Desarrollo: Estrategia Nacional para la Reducción de la Pobreza de la Administración Solís Rivera (2015-2018). San José, Costa Rica: IMAS.

INDER. 2012. "Ley n. 9036. Transformación del Instituto de Desarrollo Agrario (IDA) en el Instituto de Desarrollo Rural (Inder)". Diario Oficial La Gaceta 103, de 29 de mayo de 2012.

MIDEPLAN (2014). Plan Nacional de Desarrollo 2015-2018. San José, Costa Rica: MIDEPLAN, 2014.

MIDEPLAN. 2019. Plan Nacional de Desarrollo y de Inversión Pública del Bicentenario 2019-2022. San José, Costa Rica: MIDEPLAN, 2014.

PEN/CONARE. 2018. Programa Estado de la Nación en Desarrollo Humano Sostenible. Informe estado de la nación 2018. San José, C.R.: Servicios Gráficos AC, 2018.

Sáenz Segura, F., y Chaves Moreira, J. M. 2013. La Institucionalidad del Sector Agropecuario Costarricense: evolución y efectos sobre el desarrollo del sector. Vigésimo informe Estado de la Nación en desarrollo humano sostenible. San José, Costa Rica: CONARE.

Sáenz-Segura, F., Le Coq, J. F., \& Bonin, M. 2017. Políticas de apoyo a la agroecología en Costa Rica. En Sabourin, E. et al. (Eds). (2017). Políticas públicas a favor de la agroecología en América Latina y el Caribe. Red PP-AL: FAO

Samper, Mario. y Torrens Joao. 2015. Políticas públicas para el desarrollo de los territorios rurales. En Un recorrido por líneas locales: Aportes para políticas públicas en el sector rural de Centroamérica, el Caribe y la Región Andina, editado por Pitou van Dijck y Hans Nusselder. San José, CR: Centro de Estudios para el Desarrollo Rural (CDR).

Schejtman, Alexander, and Julio Berdegué. 2004. "Desarrollo territorial rural." Debates y temas rurales 1, RIMISP: 7-46.

SEPSA. 2015. Plan Sectorial de Desarrollo Agropecuario y Rural 2015-2018. Costa Rica: SEPSA, MAG, 2015.

SEPSA. 2017. Análisis del comportamiento del gasto público agropecuario costarricense 2016. Costa Rica: SEPSA, MAG, 2017.

SEPSA. 2018. Sector Agropecuario. Análisis del Comportamiento del Gasto Público. Agropecuario Costarricense. Año 2017. Costa Rica: SEPSA, MAG. 